\title{
Moral Objectivism Across the Lifespan
}

James R. Beebe (University at Buffalo) and David Sackris (John A. Logan College)

[Under Review]

\begin{abstract}
We report the results of a cross-sectional study that examined folk metaethical judgments in participants between the ages of 12 and 88 . We found that participants in their late teens and early twenties attributed less objectivity to ethical statements than participants in older or younger age groups. We also critically examine other recent investigations of folk metaethical intuitions and compare our results to certain well-known findings in the moral development literature.
\end{abstract}

keywords: moral psychology, metaethics, objectivism, subjectivism, relativism

Philosophers have long assumed that the vast majority of ordinary people are unrestricted moral objectivists - i.e., that they take moral claims to be objectively true or false and to apply to all people regardless of culture. For example, J. L. Mackie $(1977,33)$ famously claimed:

The ordinary user of moral language means to say something about whatever it is that he characterizes morally, for example a possible action, as it is in itself... and not about, or even simply expressive of, his, or anyone else's, attitude or relation to it... one that is absolute, not contingent upon any desire or preference or policy or choice. 
More recently, Michael Smith $(1994,6,84)$ has argued that "we seem to think moral questions have correct answers; that the correct answers are made correct by objective moral facts." and that "it is a platitude that our moral judgements at least purport to be objective."1 Despite the prevalence of these claims within the philosophical community ${ }^{2}$, however, the fact remains that they lack any empirical basis.

Recently, however, moral psychologists and experimental philosophers have begun investigating folk metaethical intuitions in a systematic fashion, and a variety of interesting results have been obtained. Geoffrey Goodwin and John Darley (2008, 1343), for example, report finding that "individuals tend to regard ethical statements as clearly more objective than social conventions and tastes, and almost as objective as scientific facts" but that there was considerable variation in metaethical intuitions across individuals and across different ethical issues. Goodwin and Darley (2012) also report (i) that participants treated statements condemning ethical wrongdoing as more objective than statements enjoining good or morally exemplary actions, (ii) that perceived consensus regarding an ethical statement positively influenced ratings of metaethical objectivity, and (iii) that moral objectivism was associated with greater discomfort with and more pejorative attributions toward those with whom individuals disagreed.

Hagop Sarkissian, John Park, David Tien, Jennifer Wright, and Joshua Knobe (2011) found that folk intuitions about metaethical objectivity vary as a function of cultural distance, with increased cultural distance between disagreeing parties leading to decreased attributions of metaethical objectivity. Jennifer Cole Wright, Piper Grandjean, and Cullen McWhite

\footnotetext{
${ }^{1}$ Indeed, what Smith calls 'the moral problem' is constituted by the following conjunction of alleged facts that do not sit well together: (i) Moral Objectivity: moral judgements are beliefs about matters of fact, (ii) Internalism: moral judgements, by themselves, motivate those who make them, and (iii) Humean Theory of Motivation: beliefs and desires are distinct states; beliefs on their own cannot motivate.

${ }^{2}$ Similar claims can be found in Brink (1989) and Darwall (1998).
} 
(forthcoming) found that not only is there significant diversity among individuals with regard to the objectivity they attribute to ethical claims; there is also significant diversity of opinion with respect to whether individuals take certain issues such as abortion or anonymously donating money to charity to be ethical issues at all, despite the fact that philosophers overwhelmingly regard these issues as ethical.

In the present article we report the results of a study that contributes to this growing body of empirically-based investigations of folk metaethical judgments. The two primary motivations for our project are that existing studies of folk metaethical judgments almost always employ problematic measures of moral objectivism and do not sample from a wide range of age groups. We show that individuals in their late teens and twenties often question or reject moral objectivism to a greater extent than at any other time in their lives. Before reporting the results of our study, we first review some of the difficulties and limitations of other studies of folk moral objectivism and explain how we attempted to overcome them in our own.

\section{Background and Motivation}

As is well known, philosophers divide the field of ethics into metaethics, normative ethics, and applied ethics. ${ }^{3}$ Some of the most important metaethical questions include:

(1.1) What is the distinction (if any) between ethical norms and social conventions?

(1.2) Does making an ethical judgment involve only the expression of one's emotions?

(1.3) Do all people have the same moral obligations or does what morality requires vary from culture to culture?

\footnotetext{
${ }^{3}$ Metaethics centers around questions about the meaning, source, grounding, or objectivity of ethical judgments. Normative ethics is the branch of ethics devoted to the search for general standards or principles that can serve as the basis for judging the rightness or wrongness of actions. Some well-known normative ethical principles include 'Respect the autonomy of every person' and 'Maximize the expected favorable consequences of one's actions.' Applied ethics focuses on the rightness or wrongness of specific kinds of actions-e.g., euthanasia, abortion, or the death penalty.
} 
(1.4) Are moral judgments objectively true or false?

(1.5) Are there moral facts, and if so, how do these facts compare to scientific facts?

Goodwin and Darley (2008) undertook an examination of folk thinking about (1.4) and (1.5), which pertain to the mind-independence or objectivity of moral claims. Before their work, they note that metaethics was "largely unexplored" or only "explored tangentially" among moral psychologists, and their work has sparked a significant amount of subsequent research.

Goodwin and Darley $(2008,1357-8)$ attempted to investigate the extent to which ordinary participants were "ethical objectivists (i.e., individuals who take their ethical beliefs to express true facts about the world)" or "ethical subjectivists (i.e., individuals who take their ethical beliefs to be mind-dependent, and to express nothing more than facts about human psychology)." The most significant conclusion Goodwin and Darley $(2008,1343)$ draw is that "individuals tend to regard ethical statements as clearly more objective than social conventions and tastes, and almost as objective as scientific facts," although they note "there was considerable variation in objectivism, both across different ethical statements, and across individuals.”

However, one difficulty with interpreting Goodwin and Darley's results is that their research materials often fail to distinguish between normative ethical judgments and metaethical judgments, and they often conflate metaphysical and epistemological issues in problematic ways. For example, in their first experiment Goodwin and Darley (2008) asked participants to select one of the following options as the best description for each of several ethical statements:

(2.1) True statement.

(2.2) False statement.

(2.3) An opinion or attitude. 
Goodwin and Darley interpreted the first two answer choices as objectivist answers, and the third one as non-objectivist. However, this task is problematic as a test for objectivism for several reasons. One is that the answer choices above conflate first-order (i.e., normative ethical) and second-order (metaethical) judgments. Participants in Goodwin and Darley's study were initially asked for their first-order opinions about cheating, discrimination, and other ethical issues - that is, they were asked about the extent to which they agreed or disagreed that the actions described were morally good or bad. In the metaethical task, participants were supposed to be directed to reflect upon their first-order opinions at a higher level (perhaps with some degree of critical distance) and to consider the objectivity of those opinions. But asking if participants think that an ethical statement is true or false (as in 2.1 or 2.2) is not asking them a metaethical question at all. It is simply asking them another first-order question.

Furthermore, Goodwin and Darley presumably intended 'opinion or attitude' to be understood as something that is neither true nor false-otherwise, the answer choices would not be exclusive, and there would be no contrast between the first two and the third. While some expressions (e.g., "Boo!" or "Hooray!") can succeed as communicative utterances without being the sort of things that are true or false, the most common or default interpretation of 'opinion' does not preclude the possession of a truth value. Many people currently have opinions about who will campaign to be the next President of the United States, and there is nothing about the fact that these opinions are opinions that prevents them from being true or false. Note, too, that we often consult 'expert opinion' or 'informed opinion' about some matters, and we do so because they think these opinions are more likely to be true than naïve or uninformed opinions. Moreover, even if it were granted that some opinions can be true or false, it would remain an open question as to whether a subjectivist or objectivist interpretation should be offered of what 
determines their truth values. ${ }^{4}$ Thus, because (2.1) and (2.2) fail to present objectivist answers at all and because (2.3) fails to clearly represent a non-objectivist one, we conclude that Goodwin and Darley's (2008) first measure of moral objectivism is adequate. ${ }^{5}$

Goodwin and Darley (2008) also employ a second measure of moral objectivism in their first experiment. Participants were asked how they would regard a situation in which someone else disagrees with them about the truth values of certain ethical statements. They were then instructed to select one of the following options for each target statement:

(3.1) The other person is surely mistaken.

(3.2) It is possible that neither you nor the other person is mistaken.

(3.3) It could be that you are mistaken, and the other person is correct.

(3.4) Other.

Goodwin and Darley interpreted the first answer choice as "fully objective" and the second and third choices as "intermediately objective." However, this second measure of objectivism also conflates distinct levels of assessment and fails to keep metaphysical and epistemological and related issues distinct. As before, participants were asked to indicate their first-order opinions

\footnotetext{
${ }^{4}$ These difficulties are compounded by the fact that Goodwin and Darley $(2008,1343)$ appear to invoke the truth-valued sense of 'opinion' when they instruct participants to "to indicate your opinion [sic] about the status of each statement - whether it is true, false, or an opinion." Conflation of metaphysical and epistemological issues can also be seen when Goodwin and Darley $(2008,1341)$ write, "Our interest centers on this second question, which concerns the source of such beliefs or standards - whether they derive their truth (or warrant) independently of human minds (i.e., objectively), or whether instead, their truth is entirely mind-dependent or subjective." Whether moral judgments are objectively true or false is a metaphysical question. What kind of warrant they enjoy, however, is an epistemological one.

5 The default interpretation of 'opinion' also seems to be neutral with respect to the epistemological question of the rational or evidential merits of opinions. The mere fact that an attitude is an opinion does not tell us whether it is well-grounded or based upon uninformed prejudice or superstition. That being said, there is a common, non-neutral use of 'opinion' that is generated when someone's point of view is said to be 'merely an opinion,' implying that the judgment in question is not based upon good reasons or evidence. And there is a colloquial sense of 'true' and 'false' (to which philosophers strongly object) that can serve as a foil to this sense of 'opinion'—viz., one that takes 'true' and 'false' to be equivalent to 'well-confirmed' or 'disconfirmed.' On this epistemic interpretation of 'true', 'false,' and 'opinion,' the answer choices represented in (2.1) through (2.3) are asking participants to say something about the evidential merits of the ethical judgments in question. However, this is not what Goodwin and Darley hope to be investigating. Rather, they want to know whether ordinary individuals think that ethical judgments - regardless of how well or poorly confirmed they may be - have mind-independent truth values.
} 
about various ethical statements before completing a metaethical task that was supposed to direct them to make higher order judgments. Answer choice (3.1), however, does not unambiguously lead participants to reflect at the metaethical level. Suppose you say that you believe that $p$ is true, and we tell you that someone else believes that $p$ is false. If we then ask you whether the other person is mistaken, this may simply be testing your ability to reason from ' $p$ is true' to " $p$ is false' is false' - all of which can remain at the first-order level. Goodwin and Darley were obviously hoping that anyone selecting (3.1) would be trying to express their commitment to objectivism, but the answer choice does not make this the only or even the most natural available interpretation.

An additional problem with (3.1) concerns the fact that it uses the term 'surely.' In the first-order task that Goodwin and Darley gave to participants, the answers choices included two components - one concerning valence and another concerning confidence. Participants were supposed to say whether they agree or disagreed with certain ethical statements and at the same time to indicate how strongly they agreed or disagreed. However, in the metaethical task participants were supposed to make a judgment that had nothing to do with the strength of their first-order opinions. Yet the term 'surely' is most naturally understood as expressing a strong degree of confidence and as modifying participants' first-order judgments. Mary thinks that Joe is surely mistaken if Mary is quite confident that he is. If Mary is a moral objectivist, Mary will be confident that there is a correct answer to the question of whether the target statement is true or false. But 'The other person is surely mistaken [in their first-order opinion]' does not capture this higher-order commitment very well. ${ }^{6}$

\footnotetext{
${ }^{6}$ Goodwin and Darley $(2008,1344)$ take themselves to be following other moral philosophers by viewing "one of the hallmarks of ethical objectivism to be the implication that, in cases of genuine moral disagreement..., at least one of the parties must be mistaken." Their error seems to stem from taking the ontic modal 'must' to be equivalent to the subjective 'surely.'
} 
Option (3.3) similarly includes an expression (viz., 'it could be') that is most naturally interpreted as concerning the degree of confidence that participants have in their first-order opinions. Many philosophers (e.g., DeRose 1991, Egan and Weatherson 2011, inter alia) contend that statements like the one in (3.3) are best interpreted as expressions of epistemic possibility-i.e., as expressing something like 'For all I know, $p$ is true.' From this perspective, (3.3) is best interpreted as indicating that an individual holds an opinion but does not think she possesses enough evidence to be supremely confident that it is correct. Again, however, issues concerning confidence, evidence, or certainty are distinct from the metaphysical issues that lie at the heart of the kind of objectivism Goodwin and Darley intend to be investigating.

Goodwin and Darley (2008, 1341-2) would have been better served by following more directly their own account of objectivism:

[A]lthough there are a variety of ways that philosophers have distinguished objectivism and subjectivism, one simple and respectable formulation is as follows: if an individual takes a particular ethical claim to be true, and regards situations of ethical disagreement as necessarily implying that at least one party is mistaken, then they are an objectivist (with respect to that statement), whereas if they instead allow that neither party need be mistaken, then they are a subjectivist.

In other words, if they had simply asked whether it is possible for both disagreeing parties to be correct or whether at least one of them must be mistaken, they would have had a better probe for the kind of objectivism that was their target. In the study that we report below, we direct our participants to respond in precisely this fashion. ${ }^{7}$

${ }^{7}$ Perhaps in response to worries such as those raised above, in their second experiment Goodwin and Darley $(2008$, 1350) replaced the 'true, false, or opinion' question discussed above with 'Can there be a correct answer as to whether the statement in question is true?' Participants were given the answer choices 'Yes' and 'No.' However, Goodwin and Darley (2008) continued to include the four options about who is mistaken (i.e., 'The other 
In more recent work, Goodwin and Darley (2012) use a new measure of moral objectivism that suffers from the same kinds of problems that afflict their previous measures. After telling participants that there was at least one other person in the study who disagreed with them about each of several ethical statements, participants were asked to indicate "the extent to which they thought the disagreeing other person was mistaken, as opposed to neither party being mistaken" on a six-point scale that ranged from 'Neither of us need be mistaken' to 'Other person is clearly mistaken.' Again, however, first- and second-order judgments are not kept clearly distinct. Thinking that the other person is mistaken may simply mean that one has a firstorder opinion about the matter. It may not by itself imply anything about one's metaethical position. In addition, Goodwin and Darley once again include the term 'clearly' in what is supposed to be a second-order metaethical judgment, even though the term seems most naturally interpreted in this context as an expression of confidence in one's first-order moral opinion.

Other measures of metaethical objectivity suffer from significant difficulties as well. For example, Shaun Nichols $(2004,7)$ attempted to measure undergraduates' commitment to moral objectivism by asking them which of the following options best characterized a situation in which two people disagreed about the moral permissibility of hitting someone just because you feel like it:

(4.1) It is okay to hit people just because you feel like it, so John is right and Fred is wrong.

(4.2) It is not okay to hit people just because you feel like it, so Fred is right and John is wrong.

person is surely mistaken,' 'It is possible that neither you nor the other person is mistaken,' etc.) in their second experiment. And in their third experiment they asked participants what they would conclude about someone who hypothetically disagreed with them, directing them to use an answer scale that ranged from 'neither of us need be mistaken' to 'the other person is clearly mistaken.' Again, however, 'the other person is clearly mistaken' cannot be a pure measure of objectivism insofar as it incorporates an element of subjective confidence. 
(4.3) There is no fact of the matter about unqualified claims like "It's okay to hit people just because you feel like it." Different cultures believe different things, and it is not absolutely true or false that it's okay to hit people just because you feel like it.

However, these answer choices fail to serve as an adequate measure of objectivism. Options (4.1) and (4.2) simply ask participants to report their first-order opinions about the morality of hitting someone for no good reason. In other words, these answer choices do not ask participants to make metaethical judgments at all. The third choice does concern metaethical issues, but it fails to serve as a good foil to the first two. Nichols seems to be assuming that someone cannot be a non-objectivist and at the same time think that Fred is right and John is wrong (or vice versa) because participants are forced to choose between giving a non-objectivist answer and saying that one of the parties is mistaken. But moral non-objectivists do not cease to have moral opinions when they reject objectivism. They simply do not think those opinions are objectively correct or incorrect.

In the same article, Nichols $(2004,19)$ used the following set of answer choices as another measure of moral objectivism:

(5.1) It is an objective fact, independent of what different people think, that it was not wrong for Frank to hit Ben and for Lisa to shove Nancy. So John is right and Ted is wrong.

(5.2) It is an objective fact, independent of what different people think, that it was wrong for Frank to hit Ben or for Lisa to shove Nancy. So Ted is right and John is wrong.

(5.3) There is no objective fact, independent of what different people think, about whether it was wrong for Frank to hit Bill or Lisa to shove Nancy. These actions 
were 'wrong for Ted' and maybe 'wrong for me,' but they aren't objectively wrong independent of what people think about them.

In addition to the fact that these answer choices jointly contain 118 words, they are also problematic because they fail to distinguish first- and second-order moral judgments. Options (5.1) and (5.2) — but not (5.3) —ask participants to make a first-order judgment about whether John's or Ted's opinion is correct and also to make a second-order judgment about the objectivity of the claims involved. First- and second-order judgments, however, are best kept distinct. And because (5.3) — like (4.3) above — only concerns second-order (i.e., metaethical) matters, it again precludes participants from registering both a non-objectivist metaethical judgment and a first-order judgment about hitting.

In another study, Cecilia Wainryb, Leigh Shaw, Marcie Langley, Kim Cottam, and Renee Lewis (2004) presented five-, seven- and nine-year old children with two cases of moral disagreement, one of which is "Sarah believes that it's okay to hit and kick other children, and Sophie believes that it's wrong to hit and kick other children" They asked participants, "Do you think that only one belief is right, or do you think that both beliefs are right?" Children who stated that only one belief is right were then asked, "Which one is right?" The question 'Do you think that only one belief is right...' is supposed to be a second-order, metaethical question. However, it comes close to confusing first- and second-order issues in ways that are similar to the probes of Nichols (2004) and Goodwin and Darley (2008). If a child indicates that she thinks only one belief is correct, this is most naturally interpreted as indicating that she has a first-order moral opinion about hitting and kicking other children. What can be inferred about a child's second-order metaethical beliefs from the fact that she has a first-order moral opinion? Nothing, it seems. For those children who select the second disjunct of Wainryb et al.'s first question (i.e., 
'both beliefs are right'), this may well tell us something about their metaethical beliefs. But since the first disjunct does not clearly do so, the disjunctive question as a whole fails to constitute an ideal measure of metaethical commitments. ${ }^{8}$

Some scholars (e.g., Nucci 2001, Nichols \& Folds-Bennett 2003, Nichols 2004, inter alia) mistakenly take Elliot Turiel's (1983) work on the moral/conventional distinction to involve the study of folk moral objectivism. For example, Nichols and Folds-Bennett (2003, B23) contend that "the child's capacity to distinguish morality from convention shows that children regard moral violations as objectively wrong." However, the fact that children treat moral violations (e.g., hitting someone) as more generalizable, more serious, and less contingent on the decrees of particular individuals in authority than conventional violations (e.g., speaking in class without raising one's hand) does not show that they are moral objectivists. The results concerning seriousness and contingency may only show that children recognize that some socially constructed, mind-dependent norms are more important than others. As Goodwin and Darley $(2010,167)$ note, these data show only that moral claims on the whole have a deeper warrant that conventional ones, but that "this deeper warrant could be either subjective or objective-it could arise from the subjective mental states of members of a community (subjective), or it could arise from an external source, such as God (objective), or from some other objective source." Goodwin and Darley (ibid.) also point out that Turiel's data about

\footnotetext{
${ }^{8}$ Nichols and Folds-Bennett (2003) also investigated the metaethical judgments of young children. They employed a measure of preference-dependence that asked four- to six-year-old children whether moral properties like 'good' and 'bad' and response-dependent properties like 'yummy,' 'icky,' and 'fun' were yummy (or good, bad, etc.) 'for some people' or 'for real.' According to Nichols and Folds-Bennett (2003, B30), the results they obtained "suggest that children are indeed moral objectivists." However, they found that children attribute the same degree of preference-independence to conventional transgressions (e.g., standing during story-time or drinking soup out of a bowl) that they do to moral transgressions. Yet Nichols and Folds-Bennett conclude from this data that children are objectivists about morality but not that they are objectivists about conventions. Nichols and Folds-Bennett do not adequately explain why the same inference is not warranted in both cases.
} 
whether children think an action would still be right or wrong in another culture concern the scope of moral claims rather than their objectivity.

Somewhat surprisingly, after claiming that Turiel's work on the moral/conventional distinction reveals children to be moral objectivists, Nichols (2004, 20-12) reports that undergraduates who reject objectivism still treat moral claims as different from conventional ones:

On the standard version of the moral/conventional task used here, students responding as moral nonobjectivists about standard moral violations drew the moral/conventional distinction just like objectivists, and indeed, there is little to distinguish objectivists from nonobjectivists in any of these experiments.

But if objectivists and non-objectivists both draw the moral/conventional distinction, then drawing it should not be taken as evidence that one is an objectivist.

Because of the difficulties with extant measures of moral objectivism reviewed in this section, we thought there was a need to develop and employ a cleaner and less problematic measure. As we describe below, our research materials drew an explicit separation between the task of indicating one's first-order moral opinion and the task of making a metaethical judgment. For the latter task, we asked participants, "If someone disagrees with you about whether [a moral statement is true], is it possible for both of you to be correct or must at least one of you be mistaken?"

A second motivation for our project came from the limited age range found in existing studies of folk metaethical judgments. We are not aware of any published studies that examine the metaethical judgments of individuals across the entire lifespan. Comparing the non- 
objectivism of the undergraduates in his studies with the supposed moral objectivism of children, Nichols $(2004,11)$ writes:

If, as seems possible, some of these college students fully reject moral objectivism, it generates the longitudinal result that, at some point in their development, a number of individuals convert from objectivism to nonobjectivism. ${ }^{9}$

Because the data at Nichols' disposal do not go beyond the college years, they are unable to speak to the question of whether undergraduates who reject objectivism will continue to do so throughout adulthood. In order to address this question, we included within our surveys adults from every stage of life. We surveyed approximately two and half thousand participants between the ages twelve and eighty-eight and found a significant difference between the metaethical objectivity judgments of college-aged participants and participants younger and older than them.

\section{Our Study}

Goodwin and Darley $(2008,1343)$ presented participants with a variety of claims from the domains of science, morality, convention, and taste, selecting items "on the basis of pilot testing as those that tended to produce either relatively strong agreement or disagreement." In our opinion, the unforeseen result of this method of item selection was that the scientific statements tended to be significantly less controversial than the moral statements. Because Goodwin and Darley wanted to compare the objectivity ratings participants gave to scientific and moral claims, we thought it would be better if the research materials exhibited a more equal balance of controversial and uncontroversial statements within these domains. Thus, we modified some of the statements used by Goodwin and Darley and introduced others so that there was a roughly equal number of controversial and uncontroversial statements about matters of physical fact,

\footnotetext{
${ }^{9}$ Nichols $(2004,21-22)$ also writes "For the developmental evidence suggests that basically all children are objectivists about canonical moral violations like hitting. So college students who are nonobjectivists about such violations have presumably abandoned their earlier objectivist views about these transgressions."
} 
morality, and taste. We later confirmed our armchair hypotheses about how controversial these matters were with participant ratings of them. The statements that we used in our study appear in Table 1.

\section{Factual}

$1^{*}$. Frequent exercise usually helps people to lose weight.

2. Global warming is due primarily to human activity (for example, the burning of fossil fuels).

3. Julius Caesar did not drink wine on his 21 st birthday.

4. There is an even number of stars in the universe.

$5^{*}$. Humans evolved from more primitive primate species.

$6^{*}$. Mars is the smallest planet in the solar system.

7. The earth is only 6,000 years old.

$8^{*}$. New York City is further north than Los Angeles.

\section{Ethical}

$9^{*}$. Anonymously donating a significant portion of one's income to charity is morally good.

$10^{*}$. Assisting in the death of a friend who has a disease for which there is no known cure and who is in terrible pain and wants to die is morally permissible.

$11^{*}$. Scientific research on human embryonic stem cells is morally wrong.

$12^{*}$. Before the third month of pregnancy, abortion for any reason is morally permissible.

13. Cutting the American flag into pieces and using it to clean one's bathroom is morally wrong.

14*. Lying on behalf of a friend who is accused of murder is morally permissible.

15. Cheating on an exam that you have to pass in order to graduate is morally permissible.

$16^{*}$. Robbing a bank in order to pay for an expensive vacation is morally bad

17. Hitting someone just because you feel like it is wrong.

$18^{*}$. Treating someone poorly on the basis of their race is morally wrong.

$\underline{\text { Taste }}$

19*. Classical music is better than rock music.

20. McDonald's hamburgers taste better than hamburgers made at home.

21. Brad Pitt is better looking than Drew Carey.

22. Gourmet meals from fancy Italian restaurants taste better than microwavable frozen dinners.

$23^{*}$. Beethoven was a better musician than Britney Spears is.

$24 *$. Barack Obama is a better public speaker than George W. Bush.

Table 1. Factual, ethical, and taste claims used in our cross-sectional study. An '*' indicates that the test item was based at least in part on an item used by Goodwin and Darley (2008). 
Statements (2) and (7)—about global warming and the age of the Earth—were introduced to increase the number of controversial physical statements. We also introduced two factual or scientific statements (3 and 4) whose truth values are presently unknowable, in order to see if unknowability affected participants' objectivity ratings. Even no one presently knows whether Julius Caesar drank wine on his twenty-first birthday, there would seem to be a fact of the matter that objectively determines whether he did or not do so. We later realized that we had made an error with statement (4), since cosmologists take the universe to be spatially infinite, despite the fact that the standard Big Bang model assumes temporal finitude.

Ethical statement (13) is taken from Jonathan Haidt's (Haidt et al. 1993) work on the respects in which different populations of people take harmless but disrespectful actions to be morally wrong. Statement (17) is a standard test item in the moral/conventional literature. In the domain of taste, we wondered whether participants' might treat as objective statements that involved extreme comparisons-e.g., those between Beethoven and Britney Spears (23) and Brad Pitt and Drew Carey (21) - that (in our minds at least) seemed relatively clear-cut and fairly objective. ${ }^{10}$ We wanted to see whether-in spite of the fact that everyone feels the need to agree that physical attractiveness lies purely in the eye of the beholder - the fact that Brad Pitt would be unanimously agreed to be far better looking than Drew Carey would lead participants to attribute objectivity to statement (21). Upon further reflection, we concluded that statement (24) was not a great example of a taste statement, insofar as Bush's public speaking skills are most often associated with his occasional departures from the rules of English grammar and semantics,

\footnotetext{
${ }^{10}$ For the uninitiated, Britney Spears is an American pop singer, Brad Pitt is an American actor known for his good looks, and Drew Carey is an American comedian and television personality who is known for his quirky looks that few would describe as handsome.
} 
and these lapses are more objective than aesthetic flaws of his speech. ${ }^{11}$

Each participant was asked to complete three tasks with respect to one-third of the statements represented in Figure 1. Task 1 was to indicate the degree to which they agreed or disagreed with the statements on a six-point scale, where ' 1 ' was anchored with 'Strongly Disagree' and ' 6 ' with 'Strongly Agree.' In Task 2, participants were asked, 'If someone disagrees with you about whether [one of the test statements is true], is it possible for both of you to be correct or must one of you be mistaken?" and were directed to choose between the dichotomous answers 'It is possible for both of you to be correct' and 'At least one of you must be mistaken.' The answer 'At least one of you must be mistaken' was taken to be an attribution of objectivity, and the answer 'It is possible for both of you to be correct' was interpreted as a denial of objectivity. In Task 3, participants were asked about the extent to which they thought that "people in our society" disagreed about the statements in question. Participants registered their opinions on a six-point scale anchored with 'There is no disagreement at all' and 'There is an extremely large amount of disagreement.' Each participant completed Task 1 with respect to eight of the items in Table 1, then completed Task 2 with respect to those same items, and then completed Task 3 .

Participants were 2,527 individuals (average age $=50,43 \%$ female, $88 \%$ AngloAmerican) residing in the Buffalo, New York, metro area or having a connection to the University at Buffalo (a large, state-funded institution), ranging in age from 12 to 88. Our sample included 120 seventh graders (average age $=13$ ), 120 ninth graders (average age $=15$ ) and 120

\footnotetext{
${ }^{11}$ One concern about our research materials stems from the fact that our factual and ethical statements were non-comparative, asking participants to consider one object or event at a time, while our taste claims were comparative, asking participants to consider pairs of items. Any significant difference we obtain between participant assessments of these statement categories could well be due to differences of formulation rather than the core features of the statement categories themselves. Cova and Pain (2012), however, recently completed a study of the objectivity attributions of ordinary participants made to aesthetic statements. Using non-comparative statements that included the French predicates 'beau' (beautiful) or 'laid' (ugly), they obtained results equivalent to the ones we report below. Thanks to Carolyn Korsmeyer for pressing this point with us.
} 
twelfth graders (average age $=17$ ) from the Orchard Park School District, a middle-class suburb of Buffalo. Although lacking in significant ethnic diversity, this district was chosen because the mean and median income levels of families within it closely matched the mean and median incomes of families across the region. 120 undergraduates at the University at Buffalo and Erie County Community College (average age $=19$ ) were also included in the study. The remaining adults were primarily professional staff (no faculty ${ }^{12}$ ) from the University at Buffalo and Erie County Community College and alumni from the University at Buffalo.

We formulated several hypotheses about what we expected to see in the data. First and most importantly, we hypothesized that undergraduates between the ages of 18 and 22 and some individuals lying just outside this range would attribute less objectivity to moral statements than either younger or older participants. We also hypothesized that across all age groups there would be a negative correlation between the degree of controversy associated with a statement and the objectivity that was attributed to it. Thirdly, we hypothesized that the least controversial ethical statements would be viewed as significantly more objective than the most controversial factual statements. Our fourth hypothesis was that participants would attribute a comparatively significant degree of objectivity to statements (21) and (23). Finally, we hypothesized that the unknowability of statements (3) and (4) would significantly dampen attributions of objectivity. This latter hypothesis was associated with our desire to test the degree to which our measure of objectivity was sensitive to features other than perceived mind-independent objectivity.

The proportion of objectivist responses participants gave to each of the twenty-four statements that appear in Table 1 are represented in Figure 1. As can be seen from Figure 1, the

\footnotetext{
${ }^{12}$ Faculty were excluded because we were trying to investigate the metaethical judgments of 'the ordinary person,' and faculty fail to be ordinary in a number of ways. The most important is that their metaethical commitment are significantly more likely to be driven by theoretical considerations, whereas we wanted to investigate individuals' pretheoretical intuitions.
} 
items in Table 1 are ordered within each subcategory in terms of increasing proportions of objectivity attributions.

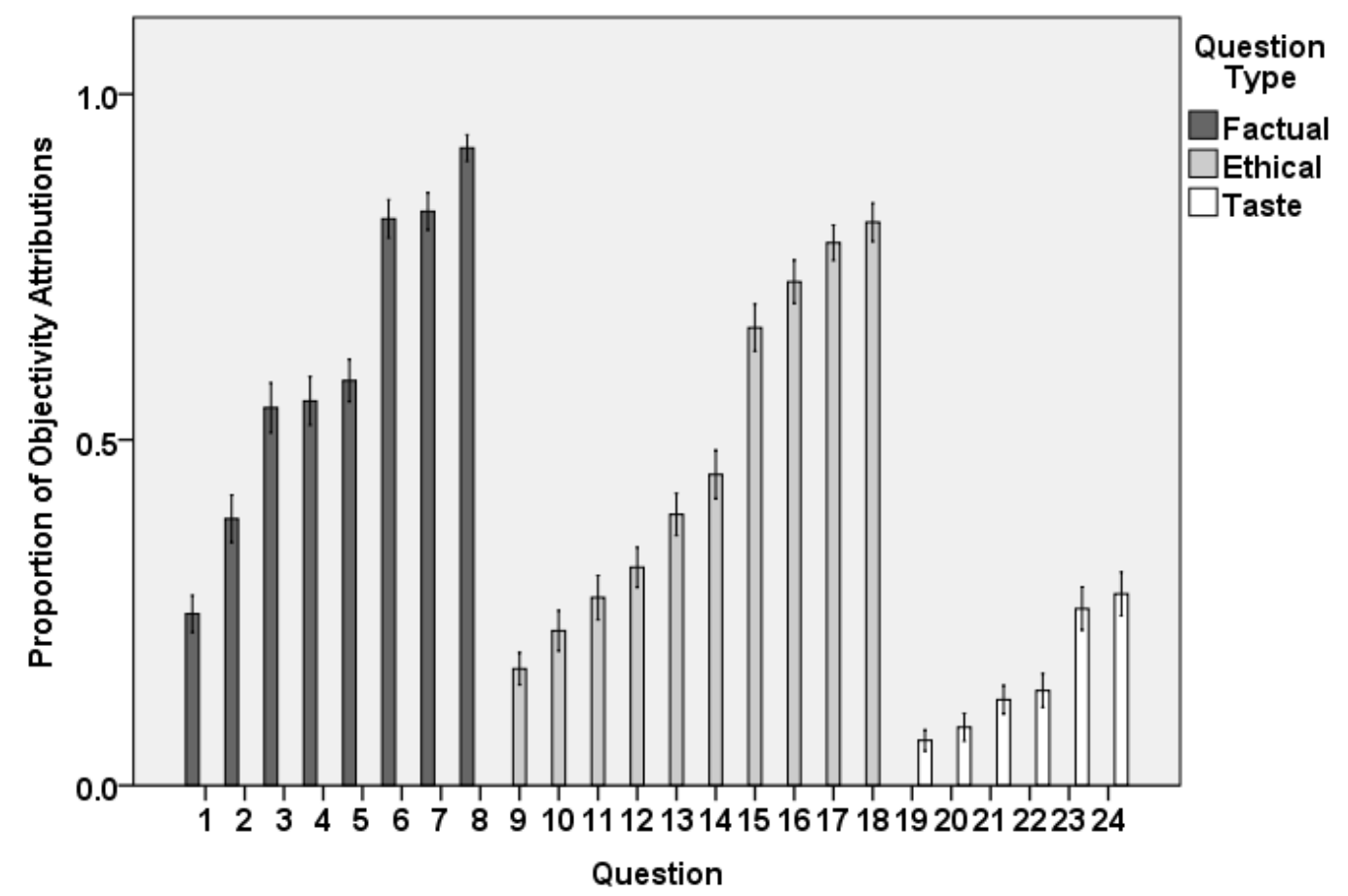

Figure 1. Proportions of participant objectivity attributions to the factual, ethical, and taste statements that appear in Table 1. Error bars in all figures represent $95 \%$ confidence intervals.

Collapsing the responses within each statement category reveals that significantly more participants attributed objectivity to factual claims (.60) than to ethical claims (.47), and more attributed objectivity to ethical claims than to taste claims (.15). ${ }^{13}$ These facts remain true even when we control for participants' strength of opinion. However, as Figure 1 clearly shows, there was significant variation in the proportion of objectivity responses given to different claims

${ }^{13}$ Factual vs. ethical: $\chi^{2}(1, N=15,054)=225.698, p<.001$, Cramér's $\mathrm{V}=.12$ (small effect size). Ethical vs. taste: $\chi^{2}(1, N=13,525)=1,427.017, p<.001$, Cramér's $\mathrm{V}=.33$ (medium effect size). 
within each subcategory. ${ }^{14}$ Within the factual domain, the statements that garnered the highest proportion of objectivist responses were those about the size of Mars (.82), the age of the Earth (.83), and the relative location of New York City and Los Angeles (.92). In the ethical domain, participants were most confident that at least one disagreeing party had to be wrong in cases that concerned robbing a bank (.73), hitting someone (.78), and racial discrimination (.81).

More participants attributed objectivity to some ethical claims than to some factual claims. For example, participants were on the whole more confident that someone had to be wrong in a disagreement about racial discrimination, robbing a bank, or hitting someone than in a disagreement about global warming (.39) or human evolution (.59). Participants were also more likely to think there was an objective answer about the comparative merits of Beethoven and Britney Spears (.26) than they were to think there was one about the goodness of anonymously donating money to charity (.17). Participants' response to the Beethoven and Britney statement was roughly equivalent to their euthanasia response (.22), and not as many participants thought there was an objective fact about Brad Pitt and Drew Carey's looks (.12) as we had anticipated.

Thus, without any further analysis of the data, we can see that because the strength of our participants' inclination toward objectivism varies according to the issue in question, the question of whether they are moral objectivists is not going to have a simple 'Yes' or 'No' answer. ${ }^{15}$ Commenting upon the variations in objectivity attributions found in their own study,

${ }^{14}$ Males also attributed more objectivity to claims in all three categories than females. Factual: $\chi^{2}(1, N=$ $6,429)=34.824, p<.001$, Cramér's V $=.07$ (small effect size). Ethical: $\chi^{2}(1, N=8,387)=7.504, p<.001$, Cramér's V $=.03$ (small effect size). Taste: $\chi^{2}(1, N=4,927)=20.954, p<.001$, Cramér's V $=.07$ (small effect size). Males also expressed more confidence about factual claims than females, but females expressed greater confidence (to a very small degree) than males about ethical claims. Factual: $\chi^{2}(2, N=6,351)=55.052, p<.001$, Cramér's V $=.09$ (small effect size). Ethical: $\chi^{2}(2, N=8,379)=11.567, p<.001$, Cramér's V $=.04$ (small effect size). Taste: $\chi^{2}(2, N=4,917)=1.135, p>.05$.

${ }^{15}$ We thus agree with Goodwin and Darley $(2010,170)$ when they write, "For instance, people tended to treat a statement about the wrongness of consciously discriminating against another person on the basis of race as 
Goodwin and Darley $(2008,1358,1359)$ claimed that "individuals were not particularly consistent in their meta-ethical positions about various ethical beliefs" and that "requirements of judgmental consistency across ethical scenarios are not considered." We think Goodwin and Darley's attribution of inconsistency to participants - an error of rationality or cognition - is uncharitable. ${ }^{16}$ When the foregoing results are combined with those of Sarkissian et al. (2011), who found that objectivist judgments decrease as two disagreeing parties become separated by increasing cultural distance, we believe the correct conclusion to draw is that any account of folk moral objectivism will need to be able to accommodate multiple dimensions of flexibility or variability. ${ }^{17}$

Within each statement category we find that objectivity attributions are positively associated with strength of belief about an issue (cf. Figure 2). ${ }^{18}$ In other words, participants attributed more objectivity to claims they had stronger opinions about. We also found that participants on the whole had stronger opinions about the ethical statements than about the factual ones. Recoding all (strong) ' 1 ' and ' 6 ' responses on Task 1 as ' 3 ,' all (moderate) '2' and ' 5 ' responses as ' 2 ,' and (weak) ' 3 ' and ' 4 ' responses as ' 1 ,' the mean strength of opinion rating was 2.20 for factual statements, 2.45 for ethical statements, and 2.22 for taste claims. The differences between these ratings were statistically significant. ${ }^{19}$ Thus, despite the positive

more objective than a statement about the goodness of anonymously donating $10 \%$ of one's income to charity. This finding contrasts with a presupposition that runs strongly through some philosophical writing - that ethical beliefs as a whole are objective or subjective, and that one's meta-ethical view should apply en masse to one's entire set of ethical beliefs, in a top-down, deductive fashion-what Sinnott-Armstrong (2009) refers to as the uniformity assumption."

${ }^{16}$ In more recent work, Goodwin and Darley $(2010 ; 2012)$ have begun to consider explanations that do not involve attributions of inconsistency but rather assume there are important differences between distinct classes of ethical statements to which ordinary individuals' metaethical judgments are sensitive.

${ }^{17} \mathrm{Cf}$. Beebe (2010) for one such account.

${ }^{18}$ Factual: $\chi^{2}(2, N=6,429)=570.377, p<.001$, Cramér's V = 30 (medium effect size). Ethical: $\chi^{2}(2, N=$ $8,463)=1,520.883, p<.001$, Cramér's $\mathrm{V}=.42$ (medium effect size). Taste: $\chi^{2}(2, N=4,970)=321.626, p<.001$, Cramér's $\mathrm{V}=.25$ (small effect size).

${ }^{19} \chi^{2}(4, N=19,963)=422.063, p<.001$, Cramér's V $=.10$ (small effect size). 
association between strength of opinion and attributions of objectivity, participants showed they were quite capable of denying objectivity to statements about which they held strong opinions.

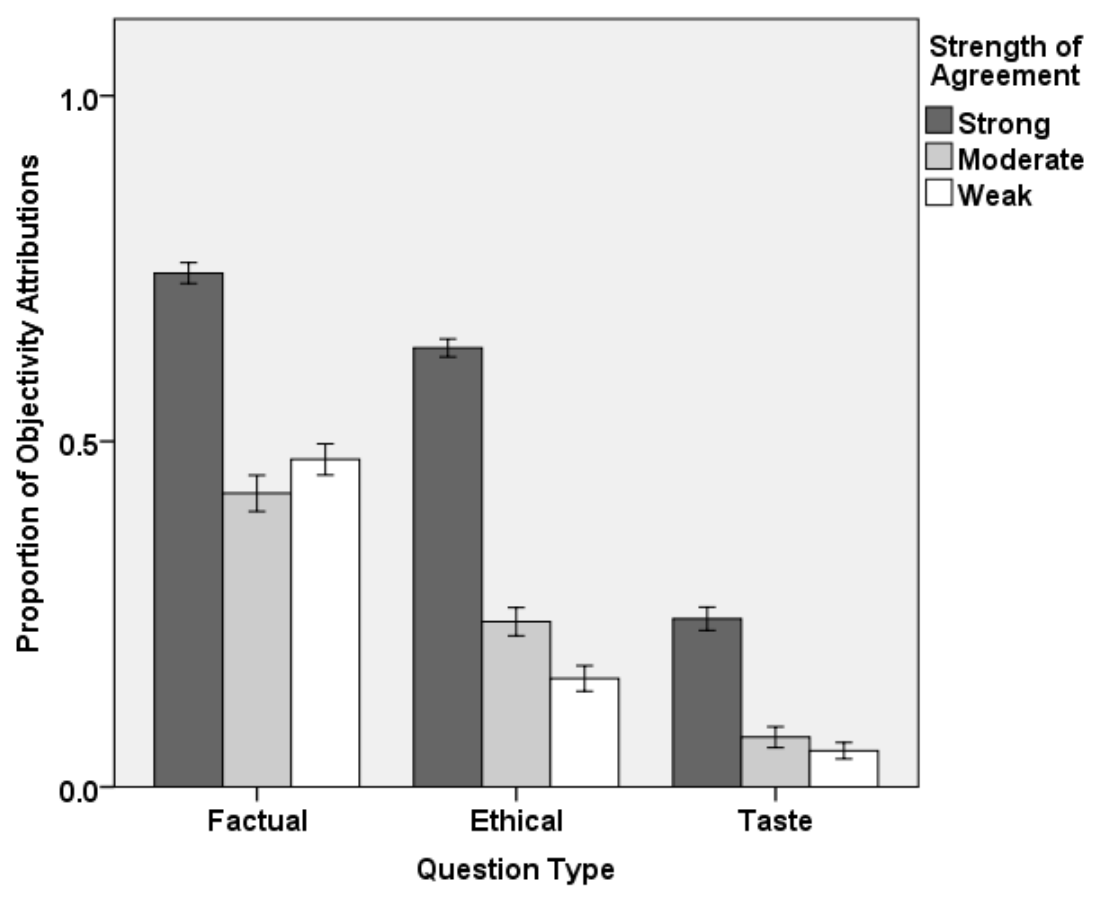

Figure 2. Proportions of participant objectivity attributions to factual, ethical, and taste statements, organized by strength of agreement.

On the whole, the average degree of perceived disagreement was higher for ethical statements (3.58) than for factual (3.29) or taste claims $(3.31) .{ }^{20}$ We also found that objectivity attributions were negatively associated with the extent of perceived disagreement about an issue (cf. Figure 3). ${ }^{21}$ The more widely disputed a statement was taken to be, the less often objectivity was attributed to it. However, despite the fact that participants rated ethical statements as being more controversial within society than taste claims, considerably more objectivity was attributed

\footnotetext{
${ }^{20}$ This difference was statistically significant. $\chi^{2}(10, N=20,003)=571.556, p<.001$, Cramér's $\mathrm{V}=.12$ (small effect size).

${ }^{21}$ Factual: $\chi^{2}(5, N=6,475)=311.905, p<.001$, Cramér's V $=.22$ (small effect size). Ethical: $\chi^{2}(5, N=$ $8,482)=731.518, p<.001$, Cramér's $\mathrm{V}=.29$ (small to medium effect size). Taste: $\chi^{2}(5, N=4,953)=312.877, p<$ .001 , Cramér's V $=.25$ (small effect size).
} 
to ethical statements than to taste claims, again showing that participants are able to separate objectivity judgments from other factors, in this case the extent of perceived disagreement. Higher ratings of perceived disagreement were also negatively associated with participants' strength of opinion about an issue. ${ }^{22}$

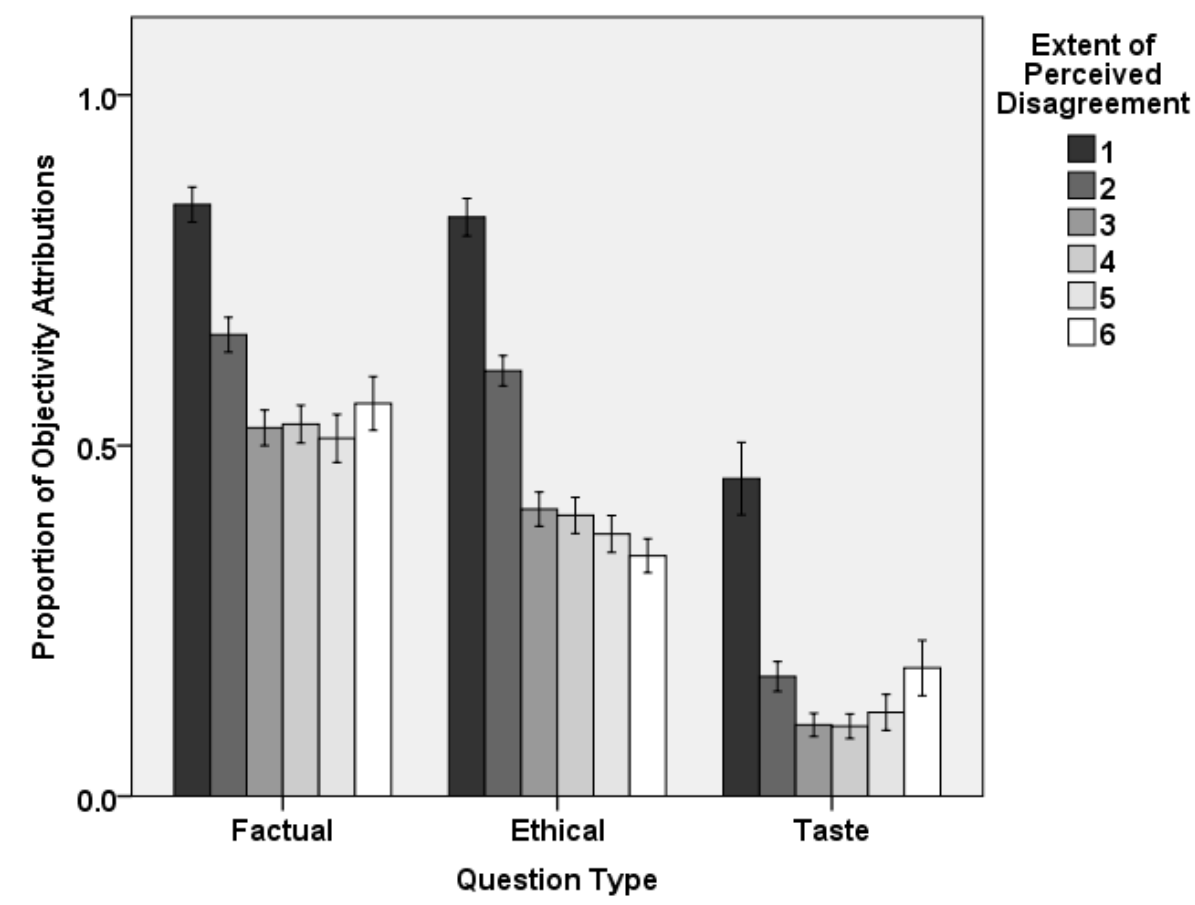

Figure 3. Proportions of participant objectivity attributions to factual, ethical, and taste statements, organized by the perceived extent of disagreement about them.

Two recent studies show that the negative association between metaethical objectivity attributions and perceived societal disagreement is more than merely correlational. Beebe (forthcoming) used the same materials employed in the present study but varied the order of Tasks 1 through 3 . Half of the participants in his study completed the tasks in the same order as above, but the other half completed Task 1, then Task 3, followed by Task 2. Having participants

${ }^{22}$ Factual: $\chi^{2}(10, N=6,413)=556.133, p<.001$, Cramér's V = .21 (small effect size). Ethical: $\chi^{2}(10, N=$ $8,469)=622.788, p<.001$, Cramér's V $=.19$ (small effect size). Taste: $\chi^{2}(10, N=4,944)=373.787, p<.001$, Cramér's V $=.19$ (small effect size). 
reflect upon the extent of societal disagreement about ethical statements (Task 3) before completing Task 2 decreased their attributions of objectivity to those statements. Notably, the task order manipulation had no effect on participants' objectivity attributions to factual or taste claims. Similarly, Goodwin and Darley (2012) presented undergraduate participants with bogus information about the percentage of students from the same institution who agreed with them about the correctness of certain ethical statements. Participants presented with low consensus estimates were significantly less likely to attribute objectivity to the statements than participants presented with high consensus estimates.

When we analyzed the association between participants' age and their attributions of objectivity, a very clear pattern emerged. Striking discontinuities were found at the boundaries between 16 and 17 years of age, 29 and 30, and 51 and 52, leading us to group participants into four age-related categories. Participants' objectivity attributions to factual, taste, and ethical statements are represented in Figures 4, 5, and 6, respectively.

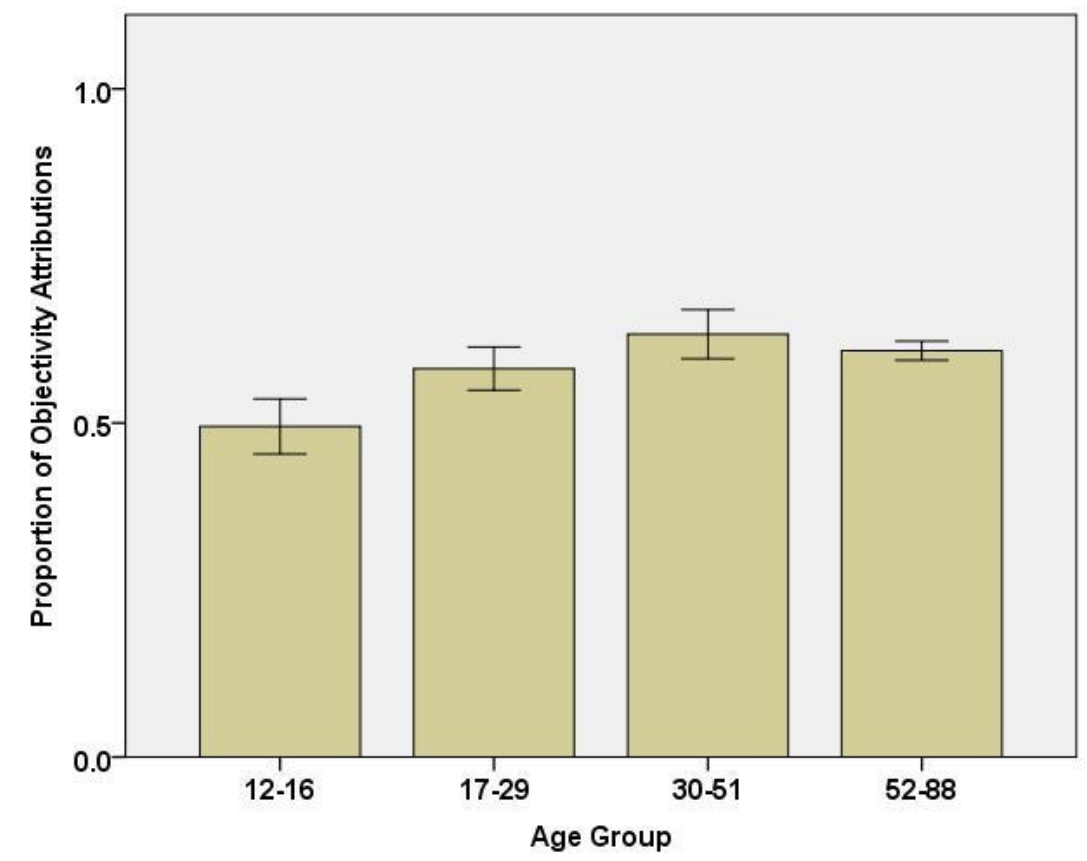

Figure 4. Proportions of participant objectivity attributions to factual statements, organized by age group. 
In Figure 4, we see that participants' confidence in the objectivity of factual or scientific statements generally increases with age. ${ }^{23}$ This seems reasonable, inasmuch as greater life experience provides one with an increased number and variety of opportunities to think about and act upon factual beliefs and to witness the reliability of scientific investigation concerning them.

Figure 5 shows that there was an overall decrease in the objectivity attributed to taste claims across the lifespan. ${ }^{24}$ Again, this seems unsurprising. While teenagers sometimes believe that their popular music and fashions really are better than the music and fashions of their parents' or grandparents' generations, older adults know that trends and fashions come and go. They know that skinny jeans will be considered fashionable for a time, and then baggy jeans will become popular. Then it will be bell-bottom jeans, and the whole cycle will repeat itself. Older individuals also tend to have greater knowledge of worldwide, cross-cultural diversity in matters of taste.

${ }^{23}$ Differences between the proportions of objectivity attributions in the factual domain are statistically significant. Analyzing the effect of the age group variable as a whole yields: $\chi^{2}(3, N=6,528)=31.521, p<.001$, Cramér's V $=.07$ (small effect size).

${ }^{24} \chi^{2}(3, N=5,001)=52.719, p<.001$, Cramér's $\mathrm{V}=.10$ (small effect size $)$. 


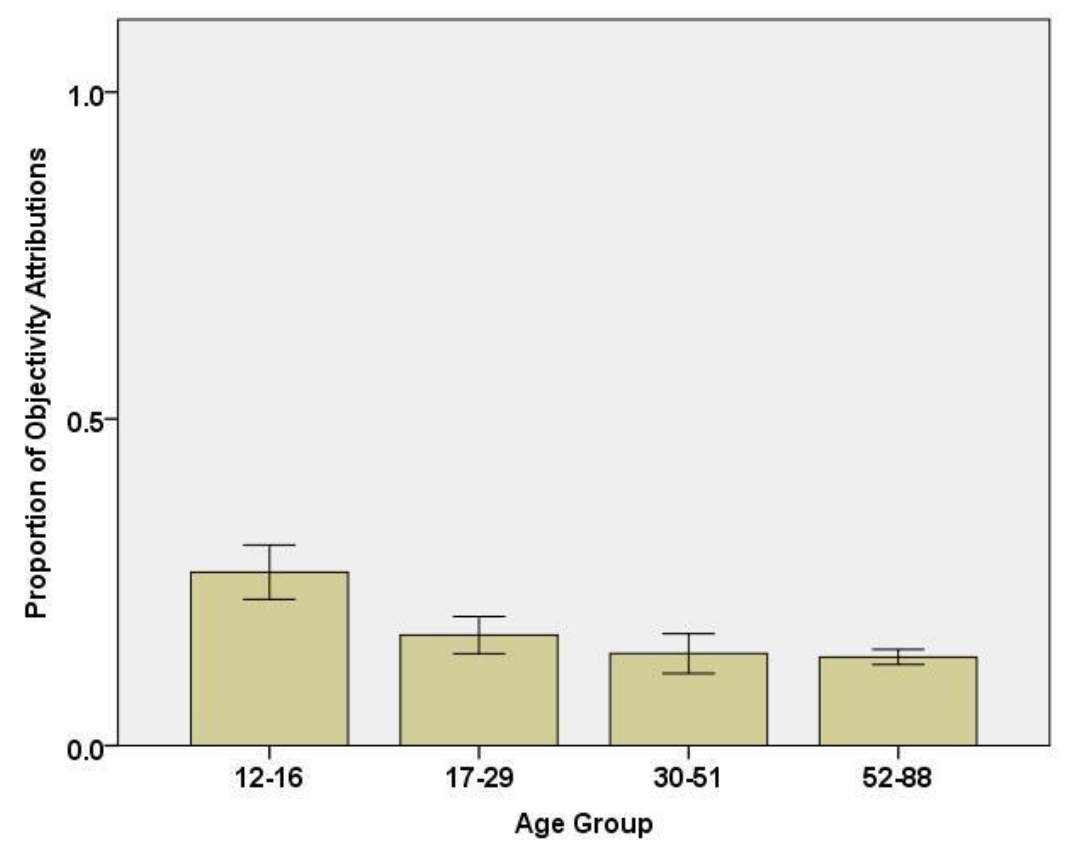

Figure 5. Proportions of participant objectivity attributions to taste statements, organized by age group.

The most significant result of the present paper is represented in Figure 6, which reveals that our prediction about folk metaethical judgments changing across the lifespan was correct. The proportion of objectivity attributions in the 17 to 29 year old group differs significantly from the proportion in the 12 to 16 year old group and those in both groups of older adults. ${ }^{25}$

${ }^{25} 12$ - 16 vs. $17-29: \chi^{2}(1, N=1,849)=19.431, p<.001$, Cramér's $\mathrm{V}=.10$ (small effect size) $.17-29$ vs. $30-51: \chi^{2}(1, N=1,962)=68.974, p<.001$, Cramér's V =.19 (small effect size). 


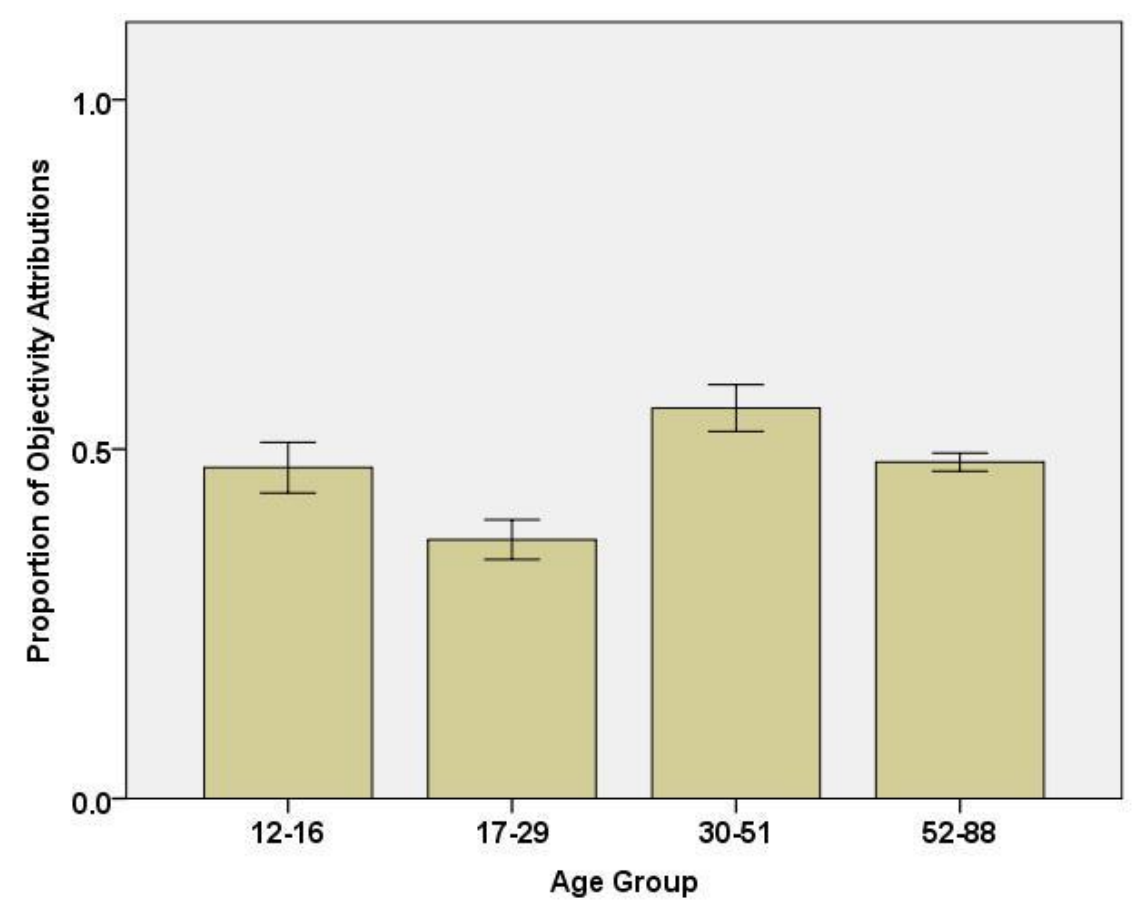

Figure 6. Proportions of participant objectivity attributions to ethical statements, organized by age group.

We predicted that we would see a significant drop in metaethical objectivism during this stage of the lifespan on the basis of several lines of reasoning. First, there is much anecdotal evidence from philosophy professors that a significant portion of their students (especially firstand second-year students) endorse some kind of non-objectivism. Secondly, we took into account the kinds of activities and experiences that participants in our sample commonly have during this stage of life. When middle-class Americans finish high school, they typically move out of their parents' home and live on their own for the first time. They enroll in college and their minds become broadened with new kinds of learning and life experiences. ${ }^{26}$ This is generally the period during which they question most intensively what their parents have always taught them

${ }^{26}$ One limitation of Study 1 is that all of our college-aged participants were enrolled in college. Working class individuals of the same age without the means to attend college will not have the same kinds of experience during this stage of life, and hence may not share the metaethical intuitions of their college-bound peers. Kohlberg $(1973,195)$ noted that none of the individuals in his longitudinal studies who did not attend college but went directly into the army or workforce exhibited the same skeptical or relativistic behavior of the sophoomre retrogressor. They also failed to attain the highest stages of moral development, according to Kohlberg. 
about morality and religion, and they decide for themselves whether to embrace or reject these teachings. Individuals in their late teens and early twenties also seem to be more open to new experiences and new ways of viewing the world that during any other period of their lives. Describing this stage of life, Lawrence Kohlberg and Richard Kramer $(1969,117)$ wrote:

Erikson has made us familiar with the fact that Western society provides the post-highschool student with a psychosocial moratorium which allows him to live out either hedonistic or morally idealistic impulses (reflected in anything from life in protest groups to life in the Peace Corps) with a freedom he has neither earlier or later in life.

Because of the cognitive openness and malleability that mark this time of life (at least among the middle-class in western societies), we hypothesized that undergraduate participants between the ages of 18 and 22 would be the least objectivist in our sample. We also hypothesized that participants who fell just outside this age range would make similar metaethical judgments. We did not, however, anticipate that the non-objectivist age range in our sample would extend all the way to 29.

Our results are consistent with the finding that openness to experience generally increases until age 30 but then significantly decreases after 30 (McCrae \& Costa, 2003; Srivastava, John, Gosling, \& Potter, 2003). Our results are also interestingly consistent with Kohlberg and Kramer's (1969) infamous findings on the 'sophomore retrogressor.' Because Kohlberg's scheme of moral development posited a pattern of "universal stepwise invariant sequences" that was irreversible, it could not handle (without modification) the fact that a sizable portion of the individuals in his longitudinal studies appeared to revert back to earlier stages of moral reasoning, even though they had allegedly already achieved higher stages. Kohlberg and Kramer $(1969,109)$ wrote: 
That paradigm of the psychological study of the normal, the college sophomore, turns out to be the oddest and most interesting moral fish of all. Between late high school and the second or third year of college, $20 \%$ of our middle class sample dropped or retrogressed in moral maturity scores.... This drop had a definite pattern.... In their college sophomore phase, they kicked both their conventional and their Stage 5 morality and replaced it with good old Stage 2 hedonistic relativism, jazzed up with some philosophic and sociopolitical jargon.

One sophomore retrogressor offered the following take on the protagonist in the well-known Heinz dilemma, who breaks into a drugstore in order to steal an overpriced drug for his ailing wife:

He was a victim of circumstances and can only be judged by other men whose varying value and interest frameworks produce subjective decisions which are neither permanent nor absolute. (Kohlberg and Kramer 1969, 110)

Kohlberg and Kramer $(1969,116,114-115)$ hypothesized that sophomore relativism may stem from the fact that "new and non-conforming patterns of thought and behavior are [being] tried out" and from "the breakdown of their expectations of a conventional moral world in the college environment":

When they left high school, they thought that people lived by conventional morality and that their rewards in life depended on living that way, too. In college, they tell us, they learned that people did not live in terms of morality, and that if they did, they weren't rewarded for it. 
According to Kohlberg, all of the individuals who engaged in non-objectivist moral thinking as they transitioned from high school to college later returned to objectivist thinking. ${ }^{27}$

After "a more careful analysis of the sense in which moral stage theory can tolerate regression" (provided by Turiel 1974), "a thorough revision of the stage-scoring system to reflect more directly the structure rather than the content of moral thought," and "a further wave of longitudinal interviews on the subjects," Kohlberg (1973, 190) eventually introduced an intermediate stage of moral development (stage 41/2) in between his fourth and fifth stages and concluded that the data on retrogressors represented true forward development rather than backsliding on the part of the subjects. While Kohlberg was concerned with the development of normative ethical judgments, and we are concerned with metaethical judgments, what is most interesting for our purposes is that Kohlberg appears to have observed the same increase in nonobjectivist thinking during early adulthood that we observed. ${ }^{28}$ This suggests that the effect we have found is developmental in nature and is not a cohort effect (i.e., an idiosyncratic feature of individuals who happen to be college-aged now but that would not characterize college-aged individuals at other times) or one that is unique to the particular set of individuals in our study.

Kohlberg and Kramer $(1969,117)$ claimed that the college student encountered new opportunities "to live out either hedonistic or morally idealistic impulses... with a freedom he has neither earlier or later in life":

${ }^{27}$ On Kohlberg's revised developmental scheme, interviewees came to be interpreted as reaching the stage of postconventional thinking at the approximate age of twenty-five at the earliest. Notice that this age corresponds roughly to the boundary we observed between non-objectivist thinking in ages $17-29$ and more objectivist thinking in older participants.

${ }^{28}$ Kohlberg later claimed that non-objectivism may even be necessary to full moral development: "We do... retain our conviction that some form of subjectivism or relativism is a necessary but not sufficient condition for movement to stage $5 \ldots$. This is because a conception of liberal tolerance and universal rights represents a stage 5 principle that presupposed a questioning of the legitimacy or absoluteness of the culture's rule system (stage 4)." (Kohlberg and Higgins 1984, 440, 441) 
This moratorium comes to an end when inner establishment of an identity or outer pressure to take responsibility in a role of work and parenthood lead the individual to a commitment to a pattern of values which 'works' within a definite social world... Neither the egoism of Stage 2 relativism of our retrogressors, nor its pretentious world-changing 'idealism' will keep a social world of responsibility for other people going. It is not, of course, that our retrogressors' moral code does not work at all, it can work to the extent of creating social movements, but it does not work if these movements are to become worlds of life-long responsibility and commitment.

A few years later, Kohlberg $(1973,196)$ offered the following, similar explanation:

This moratorium period is one of new responsibility only for the self; it is primarily an experience of attained freedom to make one's own choices for oneself. Until college, the adolescent usually lives within a world he did not make and in which the choices he must make are circumscribed. Insofar as there is movement to principled thought in the college period, it is in relation to anticipated commitment. Now free, the student seeks the moral terms or contract which he should accept in terms of future commitments. Only later, however, does the student typically have the experience of sustained responsibility for the welfare of others and the experience of irreversible moral choice which are the marks of adult personal moral experience...

These remarks not only suggest an explanation for the relativistic tendencies of many college students; they also provide grounds for expecting the relativistic stage to come to an end when adult responsibilities such as parenthood begin. On the basis of these kinds of considerations, we reasoned that participants would find being a parent (who must tell one's children how to behave) and being a non-objectivist to be more difficult than being a parent and an objectivist. 
Thus, we asked all of our adult participants to indicate whether or not they were parents. In the 17 to 29 age group, participants who were parents gave objectivist responses to ethical statements $53 \%$ of the time, whereas those who were not parents gave objectivist responses only $35 \%$ of the time. This difference was statistically significant. ${ }^{29}$ In the youngest half of the 30 to 51 age group (i.e., those aged 30 to 40), participants who were parents gave objectivist responses to ethical statements $61 \%$ of the time, compared to $52 \%$ for non-parents. ${ }^{30}$ Parenthood made no difference to metaethical objectivity attributions in participants ages 41 to 88 . We speculate that one reason why metaethical objectivity attributions were lower in the 52 to 88 age group than in the 30 to 51 age group may be that participants 52 and older tend not to have children living at home with them, whereas those in the younger group do. If the duties of child-rearing tend to push individuals toward objectivism, we might expect to see a lessening of objectivist intuitions when the process of child-rearing comes to an end and a new stage of life begins.

\section{Discussion and Conclusion}

Because most of the moral psychological research on metaethical judgments has focused on undergraduate populations (with only a few studies of children), we set out to investigate the metaethical judgments of individuals across a significant portion of the lifespan-from seventh grade through the retirement years. We chose not to include participants younger than twelve years old because we wanted to use the same research materials across all age groups and thought that individuals younger than twelve would be less able to understand the materials correctly. Our results show that researchers who wish to make developmental or longitudinal claims about folk moral objectivism need to look beyond data from children and undergraduates.

\footnotetext{
${ }^{29} \chi^{2}(1, N=1,106)=13.344, p<.001$, Cramér's $\mathrm{V}=.11$ (small effect size).

${ }^{30} \chi^{2}(1, N=513)=3.600, p=.058$, Cramér's $\mathrm{V}=.08$.
} 
One limitation of our study was that it featured a geographically, educationally, and socioeconomically restricted class of participants. However, we think this limitation is mitigated by the findings of Kohlberg and Kramer (1969) discussed above and by the findings of other researchers as well. William Perry (1970), for example, famously discovered a period of relativism about epistemological matters during young adulthood that he claimed was both transitional and tied to the college experience. More recently, Wainryb and her collaborators (Wainryb et al. 2001; Wainryb et al. 1998) studied teenagers and undergraduates and found that participants' tolerance for beliefs, speech, practices, and people with whom they disagree increased with age during these life stages. These findings provide further support for the generalizability of our results.

Another question that is raised by our study concerns the fact that statements (2) and (5) in Table 1, which concern global warming and human evolution and are highly controversial within the United States, received relatively low objectivity ratings. It seems to us that the most plausible Task 2 response for global warming skeptics and creationists to make to these statements would be to attribute objectivity to them. Creationists believe it is objectively false that humans evolved from more primitive primate species. If global warming skeptics either believe that global warming is not caused primarily by human activity or doubt that we can ever know with certainty one way or the other, it still seems that the most reasonable response for them is to think there is nonetheless a fact of the matter about the issue. But this is not what we find. Relatedly, participants were not strongly inclined to think there was a fact of the matter about whether or not Julius Caesar drank wine on his twenty-first birthday.

There are at least three possible explanations of these findings that we think deserve further investigation. The first is that any participant who offers a non-objectivist response to 
factual matters that are obviously objective is deeply confused, and this confusion prevents them from having a false (because incoherent) non-objectivist position. Nichols (2004) considers this possibility and excludes from his analysis of metaethical judgments any participant who gave a non-objectivist response to a factual statement about the physical world. However, despite the widespread endorsement of metaphysical objectivism within contemporary analytic philosophy, we think there is a very real possibility that ordinary individuals are non-objectivist about "factual" matters. Consider the prevalence of various forms of idealism in the history of philosophy and the popularity of Continental philosophy among undergraduates or the nonacademic public generally. Much to the chagrin of analytic philosophers, the average nonphilosopher who says she is interested in philosophy rarely means to be expressing an interest in problems such as perdurantism, the Gettier problem, and the sense and reference of natural kind terms. This kind of person is most often interested in the existentialists and other Continental philosophers whose outlooks are decidedly non-objectivist. Thus, a second explanation of participants' non-objectivist responses to "factual" matters is that, rather than being confused, they are accurately expressing non-objectivist metaphysical intuitions. Because we think understanding folk metaphysical objectivism or non-objectivism is as important as understanding folk metaethical objectivism or non-objectivism, we have begun to empirically investigate the former set of issues and hope to report on these findings soon.

A third possibility that we think is worthy of further investigation concerns the degree to which our preferred measure of objectivism is sensitive to things other than objectivism. We do not believe that all of the participants in our study who were led to choose a non-objectivist response to a highly contested statement did so because they believed that pervasive disagreement always indicates there is no fact of the matter. We think this is likely to be 
something that (perhaps unconsciously) influences participants' judgments some of the time. But we do not think it constitutes the whole story. Therefore, in our follow-up studies of folk metaphysical objectivism, we are trying to develop probes that are capable of distinguishing when participants are expressing judgments about mind-independent objectivity and when they are expressing opinions about other metaphysical or epistemological matters.

In any case, we believe that our study shows that folk metaethical commitments are more complex and variable than many armchair philosophers have envisioned. We expect the investigation of folk metaphysical objectivism to reveal the relevant phenomena to be multidimensional and diverse as well. 


\section{References}

Beebe, J. R. 2010. Moral relativism in context. Noûs, 44, 691-724.

Beebe, J. R. forthcoming. How different kinds of disagreement impact folk metaethical judgments. In H. Sarkissian and J. C. Wright (eds), Advances in Experimental Moral Psychology. London: Continuum.

Brink, D. O. 1989. Moral Realism and the Foundations of Ethics. Cambridge: Cambridge University Press.

Cova, F., and Pain, N. 2012. Can folk aesthetics ground aesthetic realism? The Monist, 95, 243-

63.

Darwall, S. 1998. Philosophical Ethics. Boulder, CO: Westview Press.

DeRose, K. 1991. Epistemic possibilities. Philosophical Review, 100, 581-605.

Egan, A., and Weatherson, B. (eds). 2011. Epistemic Modality. Oxford: Oxford University Press.

Goodwin, G. P., and Darley, J. M. 2008. The psychology of meta-ethics: Exploring objectivism. Cognition, 106, 1339-66.

Goodwin, G. P., and Darley, J. M. 2010. The perceived objectivity of ethical beliefs: Psychological findings and implications for public policy. Review of Philosophy and Psychology, 1, 161-88.

Goodwin, G. P., and Darley, J. M. 2012. Why are some moral beliefs perceived to be more objective than others? Journal of Experimental Social Psychology, 48, 250-56.

Haidt, J., Koller, S., and Dias, M. 1993. Affect, culture, and morality, or is it wrong to eat your dog? Journal of Personality and Social Psychology, 65, 613-28. 
Kohlberg, L. 1973. Continuities in childhood and adult moral development revisited. In P. B. Bates and K. W. Schaie (eds), Life-Span Developmental Psychology: Personality and Socialization. New York: Academic Press, pp. 179-204.

Kohlberg, L., and Kramer, K. 1969. Continuities and discontinuities in childhood and adult moral development. Human Development, 12, 93-120.

Kohlberg, L., and Higgins, A. 1984. Continuities and discontinuities in childhood and adult development revisited-again. In L. Kohlberg, Essays on Moral Development, Vol II: The Psychology of Moral Development: The Nature and Validity of Moral Stages. New York: Harper \& Row, pp. 426-97.

Mackie, J. L. 1977. Ethics: Inventing Right and Wrong. London: Penguin.

McCrae, R. R., and Costa, P. T., Jr. 2003. Personality in Adulthood: A Five-Factor Theory Perspective, 2nd ed. New York: Guilford.

Nichols, S. 2004. After objectivity: An empirical study of moral judgment. Philosophical Psychology, 17, 3-26.

Nichols, S., and Folds-Bennett, T. 2003. Are children moral objectivists? Children's judgments about moral and response-dependent properties. Cognition, 90, B23-B32.

Nucci, L. 2001. Education in the Moral Domain. Cambridge: Cambridge University Press.

Perry, Jr., W. G. 1970. Forms of Intellectual and Ethical Development in the College Years: A Scheme. New York: Holt, Rinehart and Winston.

Sarkissian, H., Parks, J., Tien, D., Wright, J. C., and Knobe. J. 2011. Folk moral relativism. Mind \& Language, 26, 482-505.

Sinnott-Armstrong, W. 2009. Mixed-up meta-ethics. Philosophical Issues, 19, 235-56.

Smith, M. 1994. The Moral Problem. Oxford: Blackwell. 
Srivastava, S., John, O. P., Gosling, S. D., and Potter, J. 2003. Development of Personality in Early and Middle Adulthood: Set Like Plaster or Persistent Change? Journal of Personality and Social Psychology, 84, 1041-53.

Turiel, E. 1974. Conflict and transition in adolescent moral development. Child Development, $45,14-29$.

Turiel, E. 1983. The Development of Social Knowledge: Morality and Convention. Cambridge: Cambridge University Press.

Wainryb, C., Shaw, L. A., and Maianu, C. 1998. Tolerance and intolerance: Children's and adolescents' judgments of dissenting beliefs, speech, persons, and conduct. Child Development, 69, 1541-55.

Wainryb, C., Shaw, L. A., Laupa, M., and Smith, K. R. 2001. Children's, adolescents', and young adults' thinking about different types of disagreements. Developmental Psychology, 37, 373-86.

Wainryb, C., Shaw, L. A., Langley, M., Cottam, K., and Lewis, R. 2004. Children's thinking about diversity of belief in the early school years: Judgments of relativism, tolerance, and disagreeing persons. Child Development, 75, 687-703.

Wright, J. C., Grandjean, P. T., and McWhite, C. B. forthcoming. The meta-ethical grounding of our moral beliefs: Evidence for meta-ethical pluralism. Philosophical Psychology. 